\title{
SPECTRAL ANALYSIS OF DATA GENERATED BY SIMULATION EXPERIMENTS WITH ECONOMETRIC MODELS ${ }^{1}$
}

\author{
By Thomas H. Naylor, Kenneth Wertz, and Thomas H. WonnacotT ${ }^{2}$
}

\begin{abstract}
This paper is concerned with the use of spectral analysis to analyze data generated by computer simulation experiments with models of economic systems. An example model serves to illustrate two different applications of spectral analysis. First, spectral analysis is used to construct confidence bands and to test hypotheses for the purpose of comparing the results of the use of two or more alternative economic policies. Second, spectral analysis is employed as a technique for validating an econometric model.
\end{abstract}

\section{INTRODUCTION}

DURING THE PAST decade computer simulation experiments with econometric models have become a commonly employed tool for analyzing the behavior of complex economic systems. While economists have improved the estimation process and have considerably enhanced the descriptive power of their econometric models, there have been fewer imposing gains made in the statistical analysis of the resulting output.

The major impetus behind the use of simulation by econometricians and economic policy makers is the possibility (and opportunity) of validating econometric models and testing and evaluating alternative economic policies before they are put into effect on actual economic systems. Complete exploitation of simulation experiments with econometric models implies a thorough analysis of the data so generated. Yet as Burdick and Naylor $[5,31,32]$ have pointed out in recent articles, a preoccupation with model building among many econometricians simulating economic systems has unduly diverted attention from experimental design considerations in general, and output analysis in particular.

Consider, for example, the monetary and fiscal policy simulation experiments with the economy of the United States described in the literature by Adelman [1, 2], De Leeuw [7], Duesenberry et al. [8], Fromm [12], and Liu [29]. Data analysis for each of these simulations was limited to either graphical or tabular presentations describing the pattern of error terms and simulated time paths of one or more endogenous variables such as $G N P$, short term interest rates, or total employment. To be sure, we have no intention of downgrading graphical and tabular analysis. But if one employs fairly high-level techniques such as two and three stage least squares to estimate the parameters of an econometric model to be used for simulation purposes, it is difficult to understand why one would be satisfied with the limited information content of rather elementary methods of output analysis.

The principal aim of this paper is to approach the problem of analyzing data generated by computer simulation experiments with econometric models. For

\footnotetext{
${ }^{1}$ This research was supported by National Science Foundation Grant GS-1104 and is a part of a collection of studies entitled "Design of Computer Simulation Experiments for Economic Systems."

${ }^{2}$ We are indebted to Mr. W. Earl Sasser of the Econometric System Simulation Program at Duke University for a number of helpful comments. Mr. David Patterson wrote the computer programs.
} 
this task we have selected "spectral analysis," a statistical technique frequently employed in the physical sciences and more recently applied by economists to analyze the behavior of economic time series $[6,13,14,15,20,34,37,38]$.

There are at least four reasons why one might want to consider spectral analysis as a technique for analyzing data generated by simulation experiments with an econometric model.

First, data generated by simulation experiments are usually highly autocorrelated, e.g., GNP in period $t$ is likely to be highly correlated with GNP in period $t-k$. It is well known that when autocorrelation is present in sample data that the use of classical statistical estimating techniques (which assume the absence of autocorrelation) will lead to underestimates of sampling variances (which are unduly large) and inefficient predictions. Several methods are available for treating this problem. (1) Simply ignore autocorrelation and compute sample means and variances over time, thereby incurring the aforementioned statistical problems. (2) Divide the sample record length into intervals that are longer than the interval of major autocorrelation and work with the observations on these supposedly independent intervals. This method suffers from the fact that "the choices of sample record length and sampling interval seem to have neither enough prior nor posterior justification in most cases to make this choice much more than arbitrary" [10]. (3) Replicate the simulation experiment and compute sample means and variances across the ensemble rather than over time. This method may lead to excessive computer running time and fail to yield the type of information that is desired about a particular time series. (4) Employ a sampling theory such as spectral analysis in which the probabilities of component outcomes in a time series depend on previous outcomes in the series. With spectral analysis the problems associated with methods (1) and (2) can be successfully avoided without replicating the experiment.

Second, "when one studies a stochastic process, he is interested in the average level of activity, deviations from this level, and how long these deviations last, once they occur" $[\mathbf{1 0}]$. Spectral analysis provides this kind of information.

Third, with spectral analysis it is relatively easy to construct confidence bands and to test hypotheses for the purpose of comparing the simulated results of the use of two or more alternative economic policies. Frequently it is impossible to detect differences in time series generated by simulation experiments when one restricts himself to simple graphical analysis.

Fourth, spectral analysis can also be used as a technique for validating an econometric model of an economic system. By comparing the estimated spectra of simulated data and corresponding real world data one can infer how well the simulation resembles the system it was designed to emulate $[\mathbf{1 0}]^{3}$

\section{AN EXAMPLE MODEL}

To illustrate the application of spectral analysis to simulation experiments with econometric models, we have chosen a stochastic version of the Samuelson-

\footnotetext{
${ }^{3}$ We return to this topic in a different context in a later section. See also $[\mathbf{3 3}, \mathbf{3 4}]$.
} 
Hicks multiplier-accelerator model $[\mathbf{2 2}, \mathbf{4 3}]$ as an example model. This model has two principal attributes. First, it is a relatively simple model and well known to economists. Second, although an analytical solution exists for certain special cases of this model, it still possesses many of the characteristics of more complex econometric models that do not lend themselves to straightforward analytical solutions. The model consists of the following parameters, variables, and functional relationships.

\section{Parameters}

(1) $\quad b$ : accelerator coefficient;

(2) $\quad c_{1}$ : marginal propensity to consume in period $t-1,0<c_{1}<1$;

(3) $\quad c_{2}$ : marginal propensity to consume in period $t-2,0<c_{2}<1$;

(4) $g:$ governmental parameter.

\section{Exogenous Variables}

(5) $\quad u_{t}$ : a stochastic variate with a known probability distribution, expected value, and variance;

(6) $\quad v_{t}$ : a stochastic variate with a known probability distribution, expected value, and variance.

\section{Endogenous Variables}

(7) $\quad C_{t}$ : consumption in period $t$;

(8) $I_{t}$ : investment in period $t$;

(9) $G_{t}$ : governmental expenditure in period $t$;

(10) $Y_{t}$ : national income in period $t$.

\section{Operating Characteristics}

(11) $f\left(u_{t}\right)$ : probability density function of $u_{t}$;

(12) $\quad f\left(v_{t}\right)$ : probability density function of $v_{t}$;

(13) $\quad C_{t}: c_{1} Y_{t-1}+c_{2} Y_{t-2}+u_{t}$;

(14) $\quad I_{t}: b\left(Y_{t-1}-Y_{t-2}\right)+v_{t}$;

(15) $G_{t}: g Y_{t-1}$.

\section{Identity}

$$
Y_{t}: C_{t}+I_{t}+G_{t}
$$

By substituting the values of $C_{t}, I_{t}$, and $G_{t}$ given by equations (13), (14), and (15) respectively into equation (16) we obtain

$$
Y_{t}=w_{t}-a_{1} Y_{t-1}-a_{2} Y_{t-2}
$$


where

$$
\begin{aligned}
& w_{t}=u_{t}+v_{t}, \\
& a_{1}=-\left(c_{1}+b+g\right), \\
& a_{2}=b-c_{2} .
\end{aligned}
$$

If we assume that national income is measured in terms of deviations $y_{t}$ from its equilibrium value, then the final form of equation (17) which determines the time path of national income is

$$
y_{t}=w_{t}-a_{1} y_{t-1}-a_{2} y_{t-2} .
$$

Equation (21) describes a second order autoregressive process. The complete analytical solution for $y_{t}$ is given by $[\mathbf{3}, \mathbf{2 3}]$ :

$$
y_{t}=k_{1} r_{1}^{t}+k_{2} r_{2}^{t}+\sum_{j=0}^{t-2} \lambda_{j} w_{t-j}
$$

where $r_{1}$ and $r_{2}$ are the characteristic roots of (21), $k_{1}$ and $k_{2}$ are arbitrary constants determined by the initial conditions, and $\lambda_{j}$ is given by

$$
\lambda_{j}=\frac{r_{1}^{j+1}-r_{2}^{j+1}}{r_{1}-r_{2}} .
$$

The solution for the time path of national income is composed of two partsa transient response and a stochastic response. The usual procedure for determining the dynamic properties of the solution of difference equation models in economics is to suppress the stochastic part of the solution and to analyze only the deterministic solution. This is equivalent to looking at the expected value of the time path of national income in our model. Philip Howrey [23] has shown that disregarding the disturbance term in the Samuelson-Hicks model may be quite misleading. He has demonstrated that stabilization policies designed to increase the stability of the system by reducing the modulus of the roots may in fact increase the variance of the system.

If our model were a simultaneous equation model (and nonrecursive), nonlinear, and of higher order than two, then analytical solutions would become increasingly difficult, and the benefits from using a computer to generate the time paths of the endogenous variables increase considerably. Although one could clearly perform experiments with our simple example model without a computer, it does serve to illustrate many of the experimental design problems which are associated with more complex econometric models involving higher order nonlinear systems of difference equations.

\section{SIMULATION RUNS}

Three separate simulation runs, each of which consisted of a sample size of two hundred, were made with the Samuelson-Hicks model. In each run $u_{t}$ and $v_{t}$ were assumed to be independent normally distributed random variables with 
expected values equal to zero and variances equal respectively to $\sigma_{u}^{2}$ and $\sigma_{v}^{2}{ }^{4}$

\section{Run 1}

The first simulation run consisted of the generation of two hundred consecutive values of $y_{t}$ using the following parameters and starting values:

$$
\begin{aligned}
c_{1} & =.375, \quad y_{0}=7.0, \\
c_{2} & =.300, \quad y_{1}=2.5, \\
b & =1.15, \\
g & =.05, \\
\sigma_{u}^{2}+\sigma_{v}^{2} & =38.26,
\end{aligned}
$$

from which

$$
\begin{aligned}
& a_{1}=-1.575, \\
& a_{2}=\quad .85, \\
& \sigma_{w}^{2}=38.26 .
\end{aligned}
$$

The output of run 1 may be considered as the result of a given monetary policy $(b=1.15)$ and a given fiscal policy $(g=.05)$ over time.

\section{Run 2}

The parameters of run 2 are identical with those of run 1 in nearly all respects; the only difference between the two runs lies in the stream of $w_{t}$ 's generated in each run. The set of $w_{t}$ 's generated in run 2 are different from those generated in run 1 even though they have the same mean, variance, and probability distribution. This result is achieved by simply changing the starting value of the pseudorandom number generator used in run 2 .

\section{Run 3}

The parameters and starting values for run 3 are given by

$$
\begin{aligned}
c_{1} & =.375, \quad y_{0}=7.0, \\
c_{2} & =.300, \quad y_{1}=2.5, \\
b & =1.05, \\
g & =.25, \\
\sigma_{u}^{2}+\sigma_{v}^{2} & =38.26,
\end{aligned}
$$

${ }^{4}$ See Chapter 4 of Naylor, Balintfy, Burdick, and Chu [31] for a collection of FORTRAN subroutines for generating stochastic variates on a computer. 
whence

$$
\begin{aligned}
& a_{1}=-1.675, \\
& a_{2}=\quad .75 .
\end{aligned}
$$

Both the monetary policy $(b=1.05)$ and the fiscal policy $(g=.25)$ used in run 3 are different from their counterparts in run 1.

\section{THEORY OF SPECTRAL ANALYSIS}

Since Granger and Hatanaka [14] and Nerlove [37] have previously presented and interpreted the theory of spectral analysis for economists, it will not be necessary to include a detailed treatment of the theory underlying spectral analysis in this paper. The definition of terms and the description of the spectral methods used to establish confidence intervals to analyze data generated by simulation experiments, however, require that we at least review some of the basic elements of spectral theory. The reader should consult the references for a complete account of the theory of spectral analysis. ${ }^{5}$

Spectral analysis considers data arranged in a series according to historical time. When one so plots the movement of many economic variables through time, he often observes a remarkable degree of smoothness in the curve; that is, although a variable assumes values that are at different times relatively high and low, its path, nevertheless, progresses evenly enough to suggest that the current value of that variable is related to its past values. In short, the data of such a time series appear to be autocorrelated. It is essentially the quantification and evaluation of this autocorrelation at which spectral analysis is aimed, after the data have been transformed into the frequency domain. ${ }^{6}$

The application of spectral analysis to a time series (whether it be actual or simulated) yields two types of information: (i) the magnitudes of deviations from the average level of a given activity and (ii) the period or length of these deviations, both of which require the transformation of the time series into the frequency domain. To obtain this information from a time series we make use of the following notation.

Denote a particular stochastic generating process or ensemble by $\left\{X_{t}, t \in T\right\}$, from which a "sample" time series $\left\{x_{t}, t=1,2, \ldots, n\right\}$ is taken. It is important to note that $\left\{X_{t}\right\}$ will indicate the manner in which $\left\{x_{t}\right\}$ is formed for all $t$; but, due to its stochastic nature, $\left\{X_{t}\right\}$ cannot determine exactly the value of the series at any particular $t$. By studying the series one attempts to approximate the structure of the generating process.

\footnotetext{
${ }^{5}$ See Blackman and Tukey [4], Granger and Hatanaka [14], Grenander and Rosenblatt [16], Hannan [18], Jenkins [24], Nerlove [37], Parzen [39], Quenouille [41], Rosenblatt [42], Tukey [44], and Wiener $[\mathbf{4 5}]$.

${ }^{6}$ There exist methods other than spectral analysis to quantify and evaluate autocorrelation, notably a parametric representation by autoregressive or moving average processes. The relevance of these methods and others to the analysis of data generated by simulation experiments with econometric models will be treated in a future paper.
} 
The generating process can be described (in part) in terms of its first and second moments : ${ }^{7}$

$$
\begin{aligned}
\mu_{t} & =E\left[X_{t}\right], \\
\sigma_{t}^{2} & =E\left[\left(X_{t}-\mu_{t}\right)^{2}\right], \\
\gamma(t, s) & =E\left[\left(X_{t}-\mu_{t}\right)\left(X_{s}-\mu_{s}\right)\right],
\end{aligned}
$$

where $E$ denotes the mathematical expectation across the ensemble, $\mu_{t}$ is the expected value (mean) of the process at time $t, \sigma_{t}^{2}$ is the variance of the process at time $t$, and $\gamma(t, s)$ is the autocovariance of the process between observations at times $t$ and $s$.

Estimation of these parameters may be accomplished if one has $M$ independent samples from $\left\{X_{t}\right\}$; i.e., $\left\{x_{t}^{k}, k=1,2, \ldots, M\right\}$. By cutting across the ensemble at $t=t_{0}$, for example, one could calculate the ensemble average estimating $\mu_{t_{0}}$ :

$$
\bar{x}_{t_{0}}=\frac{1}{M} \sum_{k=1}^{M} x_{t_{0}}^{k} .
$$

Estimates of $\sigma_{t}^{2}$ and $\gamma(s, t)$ may be obtained in a similar fashion. Although it is usually impossible to sample across the ensemble in the case of economic time series in the "real world," with computer simulation it is possible to replicate a given series by simply altering the starting value of the pseudorandom number generator used to generate the series.

We shall consider a special class of series whose first and second moments are not functions of time; i.e., there is no trend in the mean or variance of the series and its autocovariance is a function of time lag only. In symbols,

$$
\begin{aligned}
& E\left[X_{t}\right]=\mu, \\
& E\left[\left(X_{t}-\mu\right)^{2}\right]=\sigma^{2}, \\
& \begin{aligned}
E\left[\left(X_{t}-\mu\right)\left(X_{s}-\mu\right)\right] & =\gamma(t-s) \text { for all } t, s, \\
& =\gamma_{\tau},
\end{aligned}
\end{aligned}
$$

where $\tau=t-s$.

Estimates of these parameters can be obtained from a single time series by using respectively the following formulas,

$$
\begin{aligned}
\bar{x} & =\frac{1}{n} \sum_{t=1}^{n} x_{t}, \\
s^{2} & =\frac{1}{n} \sum_{t=1}^{n}\left(x_{t}-\bar{x}\right)^{2}, \\
c_{\tau} & =\frac{1}{n-\tau} \sum_{t=1}^{n-\tau}\left(x_{t}-\bar{x}\right)\left(x_{t+\tau}-\bar{x}\right),
\end{aligned}
$$

${ }^{7}$ Throughout this paper Greek letters will be used to denote parameters which characterize the stochastic process as a whole and the corresponding English letters to denote statistical estimators (random variables) based on a single time series. 
where $\gamma(0)=\sigma^{2}$ and $c_{0}=s^{2}$. Such series are sometimes called stationary in the wide sense or stationary to the second order.

The power spectrum is defined as the Fourier cosine transformation of the autocovariance:

$$
\phi(\omega)=\gamma_{0}+2 \sum_{\tau=1}^{\infty} \gamma_{\tau} \cos (\omega \tau), \quad 0 \leqslant \omega \leqslant \pi
$$

The autocovariance may be recovered from the spectrum by means of the inverse transformation:

$$
\gamma_{\tau}=\frac{1}{\pi} \int_{0}^{\pi} \phi(\omega) \cos (\omega \tau) d \omega, \quad \tau=0,1,2, \ldots
$$

For the special case where $\tau=0$ we obtain the variance as an integral of the spectrum:

$$
\sigma^{2}=\gamma_{0}=\frac{1}{\pi} \int_{0}^{\pi} \phi(\omega) d \omega
$$

Hence, we speak of the spectrum as the "decomposition" of the variance of a time series. Estimators of the power spectrum usually take the following form:

$$
f\left(\omega_{j}\right)=\lambda_{0} c_{0}+2 \sum_{\tau=1}^{m} \lambda_{\tau} c_{\tau} \cos \left(\omega_{j} \tau\right)
$$

where $f\left(\omega_{j}\right)$ is an estimate of the power spectrum averaged over a band of frequencies centered at $\omega_{j}$, and

$$
\omega_{j}=\frac{\pi j}{m}, \quad j=0,1,2, \ldots, m,
$$

$\lambda_{\tau}$ are weights,

$m$ is the number of frequency bands to be estimated.

The power spectrum gives the squared amplitude associated with oscillations at different frequencies $\omega$; that is, the process is characterized in terms of independent additive contributions to the variance as they are located at each $\omega$. Thus one plots $\phi(\omega)$ against $(\omega)$ in theory; $f\left(\omega_{j}\right)$ against $\omega_{j}$ in practice. A natural interpretation of a power spectrum is that if a band contributes a large proportion of the total variance, then it may be regarded as more important than a band where the power is less.

The selection of $m$ (number of frequency bands) and $n$ (sample size) must be made with some degree of care in order to balance the conflicting requirements of resolution and statistical stability [4]. Granger and Hatanaka [14] and Blackman and Tukey [4] have suggested some arbitrary guidelines for choosing values of $m$ and $n$. The former advise that "the amount of data required before it becomes sensible to attempt to estimate a spectrum would seem to be greater than 100," although "crude spectra have occasionally been estimated with $n$ as 
low as 80." These recommended levels would form an important barrier to investigators validating annual models whose data are of short duration. Of course, the problem is less binding for quarterly models. On the simulation side, the issue of sample size melds with considerations of experimental design, which, as is shown in the next section, guided our choice of $n=200$ and $m=25$. In an earlier study [34], we used spectral analysis (with $n=119$ and $m=24$ ) to validate a nine equation econometric model of the textile industry using monthly data between 1953 and 1962. In this case the value of $n$ was determined entirely by data availability.

To obtain empirical estimates of the power spectrum we have used a computer program developed by Karreman [26] (and modified for the IBM 360/75) which is based on the Tukey-Hanning estimator $[4,13]$.

\section{STATISTICAL PROPERTIES OF SPECTRA}

In this section we shall do four things. First, we compute the spectral estimates for the series generated by run 1 using the Samuelson-Hicks model and examine some of the properties of these estimates. Second, we formulate confidence bands for the purpose of comparing the estimated spectrum generated by run 1 with the corresponding theoretical spectrum for the Samuelson-Hicks model. Third, we formulate confidence bands for comparing the spectra of different computer runs using the Samuelson-Hicks model. Fourth, we use spectral analysis to calculate the total variance of different series generated by the Samuelson-Hicks model and formulate confidence intervals for comparing the variances of these series.

At the outset, we observe that, for our selection of parameters, assumptions (28)-(30) are satisfied for the system (21). Many economic time series, however, require intermediate treatment before spectral techniques become directly applicable. Methods for detecting and removing a trending mean as well as for handling a series whose variance and/or autocovariance changes through time are discussed at length in [14]. Although most of these methods are familiar (e.g., polynomial regression to remove a trend in mean), it must be admitted that the possible necessity of this intermediate step has probably made spectral analysis less attractive than other (but not perfectly substitutable) modes of analysis.

Without modifying our series, then, we pursue the four topics outlined for this section.

Figure 1 displays the estimated spectrum $f_{1}(\omega)$ for the series generated by run 1 using the Samuelson-Hicks model. (For convenience we use a logarithmic scale for the values of the spectrum.) The prominent peak in the empirical spectrum at $j=4$ suggests that it is this frequency $\omega_{4}$ (or one very near to it) that is making the largest contribution to the variance of the process. What further meaning is attached to this particular frequency point depends upon the time dimensions of the data. For example, suppose (i) that the output of the Samuelson-Hicks model represented monthly data and (ii) that we are interested in the annual cycle $\left(f=\frac{1}{12}\right)$. Since $\omega_{j}=j \pi / m$ and $\omega_{j}=2 \pi f$, and therefore $j=2 f m$, we may substitute 


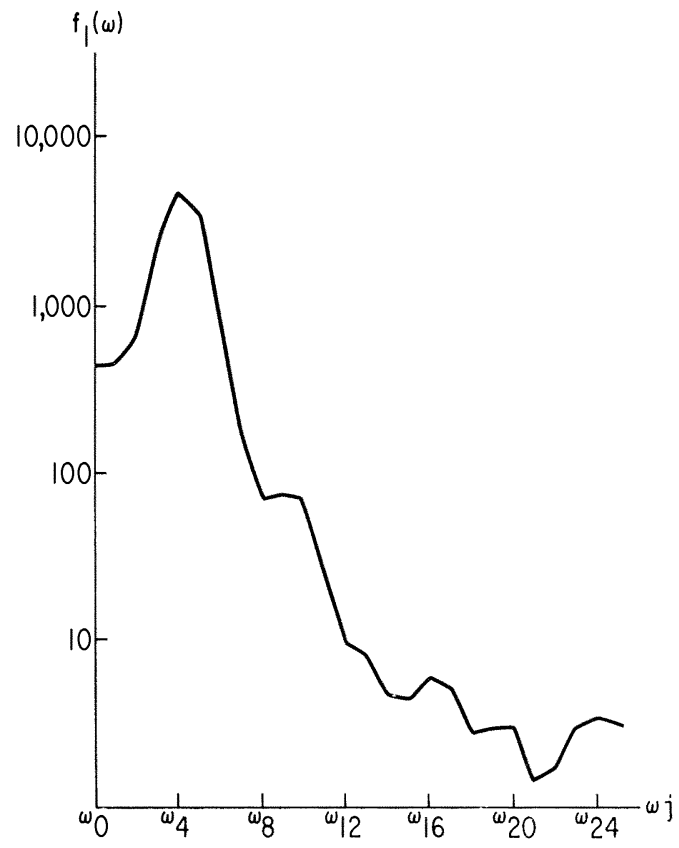

FIGURE 1.-Estimated spectrum $f_{1}(\omega)$ for the Samuelson-Hicks model (run 1).

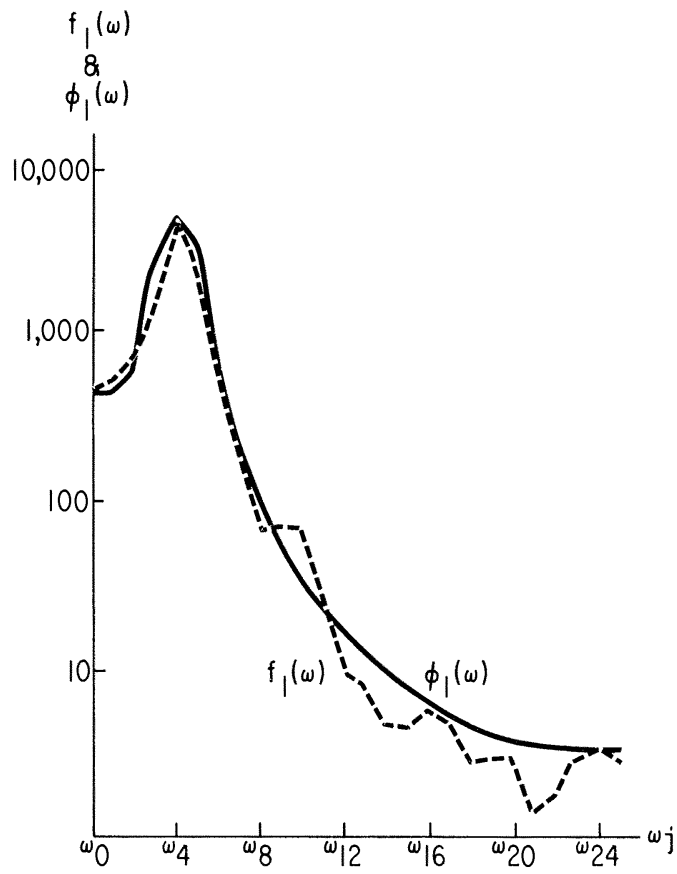

Figure 2.-Comparison of the estimated spectrum $f_{1}(\omega)$ with the theoretical spectrum $\phi_{1}(\omega)$ for the Samueison-Hicks model (run 1). 
for $f$ and $m$ in order to locate the annual component. In this example, it occurs at $j=\frac{25}{6} \simeq 4$, the approximate location, curiously enough, of the greatest power.

Figure 2 shows the theoretical and estimated spectra corresponding to run 1 of the Samuelson-Hicks model. The theoretical spectrum $\phi_{1}(\omega)$ can be calculated from the formula [23],

$$
\phi_{1}(\omega)=\frac{\sigma_{w}^{2}}{T^{2}}, \quad 0 \leqslant \omega \leqslant \pi,
$$

where,

$$
\begin{aligned}
T & =1+a_{1} e^{-i \omega}+a_{2} e^{-2 i \omega}, \\
i & =\sqrt{-1} .
\end{aligned}
$$

The coefficients $a_{1}$ and $a_{2}$ were previously defined by $-\left(c_{1}+b+g\right)$ and $\left(b-c_{2}\right)$. The shape of the spectrum is often as informative as a knowledge of the true power at a particular frequency. ${ }^{8}$ For example, Figures 1 and 2 suggest that the lower frequencies (roughly $0 \leqslant \omega \leqslant \pi / 3$ ) contribute much more to the variance of the process than do higher frequencies.

The work of Blackman and Tukey [4] informs us that spectral estimates $f\left(\omega_{j}\right)$ have desirable statistical properties, provided the process $X_{t}$ is Gaussian (normal). One property in particular, the statistical independence of spectral estimates at nonadjacent frequencies, facilitates the interpretation of confidence intervals, a method of data analysis which is familiar to most economists.

If the theoretical spectrum is reasonably smooth [4], the distribution of $f\left(\omega_{j}\right) / \phi\left(\omega_{j}\right)$ is approximately $\chi_{k}^{2} / k$ with $k=2 n / m$ degrees of freedom. ${ }^{9}$ Hence in order to gain some information about $\phi\left(\omega_{j}\right)$ when the sample $f\left(\omega_{j}\right)$ are available, one may construct a $(100-\alpha)$ per cent confidence interval for $\phi\left(\omega_{j}\right) \cdot{ }^{10}$ Suppose $\alpha=.05$; let $\chi_{.975, k}^{2}$ and $\chi_{.025, k}^{2}$ be the percentiles of the $\chi^{2}$ distribution with $k$ degrees of freedom leaving $2.5 \%$ in the lower and upper tails respectively. Then

$$
\operatorname{Pr}\left(\frac{\chi_{.975, k}^{2}}{k}<\frac{f\left(\omega_{j}\right)}{\phi\left(\omega_{j}\right)}<\frac{\chi_{.025, k}^{2}}{k}\right)=.95 .
$$

Solving for $\phi\left(\omega_{j}\right)$,

$$
\operatorname{Pr}\left(\frac{f\left(\omega_{j}\right)}{\chi_{.025, k}^{2} / k}<\phi\left(\omega_{j}\right)<\frac{f\left(\omega_{j}\right)}{\chi_{.975, k}^{2} / k}\right)=.95 .
$$

The succession of confidence intervals at the frequency points $\omega_{j}(j=0,1, \ldots, m)$, called a confidence band, is displayed in Figure 3 using the spectral estimates of the series generated by run 1 of the Samuelson-Hicks model. The theoretical spectrum $\phi_{1}(\omega)$ is also plotted in Figure 3. We would normally expect about five per cent of the confidence intervals in the confidence band to be in error.

${ }^{8}$ See $[\mathbf{1 3}]$ for an elaboration of this point.

${ }^{9}$ At the end points, $j=0$ and $j=m$, the degrees of freedom drop to $n / m$. This applies for all estimated spectra throughout the paper.

${ }^{10}$ The reader may wish to contrast the interval developed in these pages with the interval described by Granger and Hatanaka [14]. 


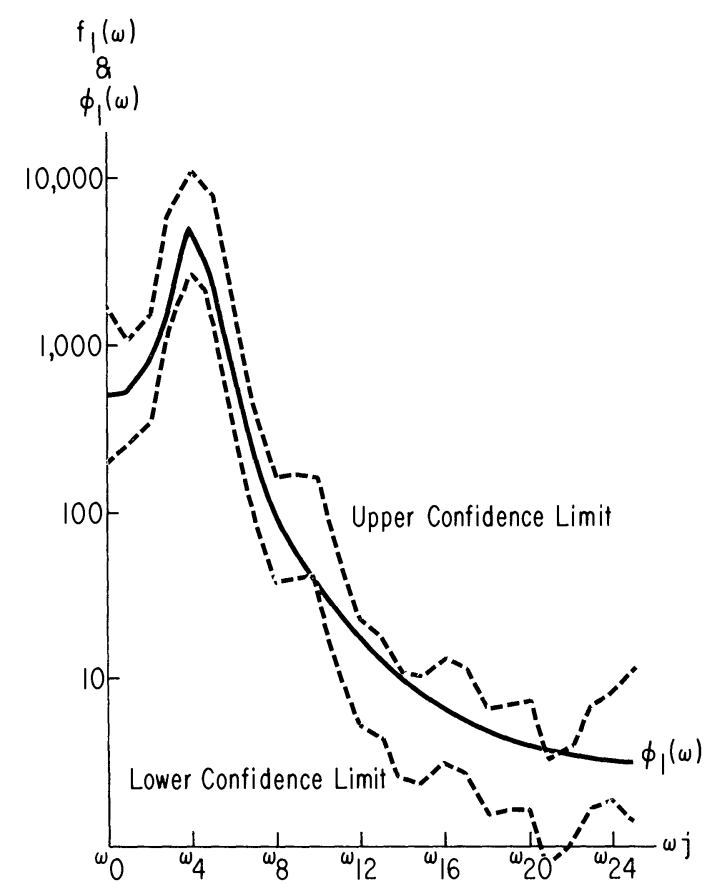

FIGURE 3.-Confidence band for spectral estimates of run 1 of the Samuelson-Hicks model.

Reference to Figure 3, where our knowledge of the theoretical spectrum is utilized, reveals that two of the twenty-six intervals $\left(\omega_{10}\right.$ and $\left.\omega_{21}\right)$, or 7.7 per cent, do not cover the true values.

The simultaneous confidence band offers a slightly different method of analysis; it permits one to state with probability $100-\alpha$ that all of the confidence intervals are simultaneously true.

Let us sketch the development of this band for $\alpha=.05 .{ }^{11}$ If $B_{j}$ denotes the "error made on the $j$ th frequency," and if we make $\operatorname{Pr}\left(B_{j}\right)=.05 /(m+1)$ $(j=0,1, \ldots, m)$, then the probability of committing any error at all is

$$
\begin{aligned}
\operatorname{Pr}\left(B_{0} \text { or } B_{1} \text { or } \ldots \text { or } B_{m}\right) & \leqslant \operatorname{Pr}\left(B_{0}\right)+\operatorname{Pr}\left(B_{1}\right)+\cdots+\operatorname{Pr}\left(B_{m}\right) \\
& =(m+1)[.05 /(m+1)] \\
& =.05
\end{aligned}
$$

The crucial inequality above does not require independence or any other hypothesis ; indeed, if the events $B_{j}$ are mutually exclusive, then the expression becomes an equality.

Hence, when we allow an error rate of $.05 /(m+1)$ for each frequency (and thus a total error rate of .05 at most $)$, then $.025 /(m+1)=.025 / 26 \simeq .001$ probability is left in each tail of the distribution. The simultaneous confidence band assumes

${ }^{11}$ An extended discussion of this proof appears in [11]. 
the same form as the confidence band developed previously, although the simultaneous band is obviously much wider than the latter. In symbols,

$$
\operatorname{Pr}\left[\frac{f\left(\omega_{j}\right)}{\chi_{.001, k}^{2} / k}<\phi\left(\omega_{j}\right)<\frac{f\left(\omega_{j}\right)}{\chi_{.999, k}^{2} / k}\right]=.95, \quad j=0,1, \ldots, m .
$$

Figure 4 depicts the 95 per cent simultaneous confidence band and the theoretical spectrum for run 1 with the Samuelson-Hicks model. This time all of the values of $\phi_{1}(\omega)$ lie within the confidence band.

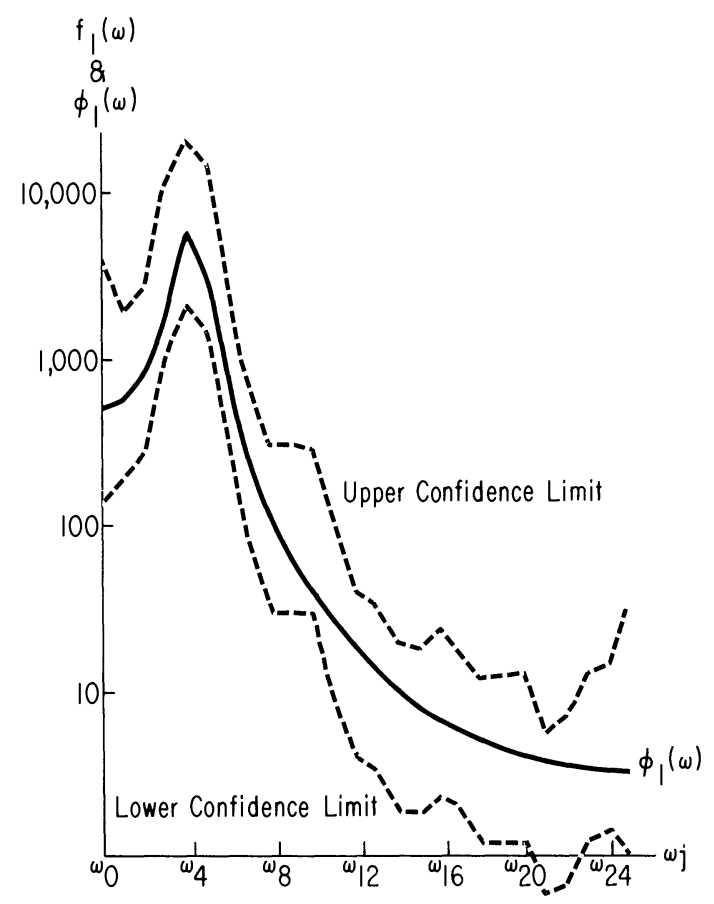

FiguRE 4.-Simultaneous confidence band for spectral estimates of run 1 of the Samuelson-Hicks model.

In the introduction to this paper we asserted that the primary interest of economists in simulation stems from the fact that with simulation it may be possible to validate econometric models and to compare the outcomes associated with alternative economic policies. Hence, economists are much more likely to be interested in constructing confidence bands to compare the estimated spectra of two series that are the result of the use of two different economic policies. Alternatively, economists may be interested in comparing the estimated spectrum of a series generated by a simulation experiment with the estimated spectrum of the corresponding "real world" series as a means of verifying the results of the simulation. 
Therefore, we next consider the comparison of two spectra via a confidence interval for the ratio $P_{j}=\phi_{1}\left(\omega_{j}\right) / \phi_{2}\left(\omega_{j}\right)$. Define $R_{j}=f_{1}\left(\omega_{j}\right) / f_{2}\left(\omega_{j}\right)$. We then obtain an $F$ (variance-ratio) statistic

$$
F_{k_{1}, k_{2}}=\frac{R_{j}}{P_{j}}=\frac{f_{1}\left(\omega_{j}\right) / \phi_{1}\left(\omega_{j}\right)}{f_{2}\left(\omega_{j}\right) / \phi_{2}\left(\omega_{j}\right)}
$$

where $k_{1}=k_{2}=2 n / m$ is the degrees of freedom for each of the two $\chi^{2}$ variates. To arrive at a 95 per cent confidence interval for $P_{j}$, let $F_{.975, k_{1}, k_{2}}$ and $F_{.025, k_{1}, k_{2}}$ be the percentiles of the $F$ distribution leaving .025 probability in the lower and upper tails, respectively. It follows that

$$
\operatorname{Pr}\left(F_{.975, k_{1}, k_{2}}<\frac{R_{j}}{P_{j}}<F_{.025, k_{1}, k_{2}}\right)=.95 .
$$

Solving for $P_{j}$, the 95 per cent confidence interval becomes

$$
\operatorname{Pr}\left(\frac{R_{j}}{F_{.025, k_{1}, k_{2}}}<P_{j}<\frac{R_{j}}{F_{.975, k_{1}, k_{2}}}\right)=.95 .
$$

Of course, a 95 per cent simultaneous confidence band may be derived for $\dot{P}_{j}$, using nearly the same reasoning employed in the development of a 95 per cent simultaneous confidence band for $\phi\left(\omega_{j}\right)$. The former may be expressed as

$$
\operatorname{Pr}\left(\frac{R_{j}}{F_{.001, k_{1}, k_{2}}}<P_{j}<\frac{R_{j}}{F_{.999, k_{1}, k_{2}}}\right)=.95 .
$$

For illustrative purposes we consider two examples. Suppose that we are interested in comparing the time path of national income generated by run 1 (again using the Samuelson-Hicks model) with the time path of national income

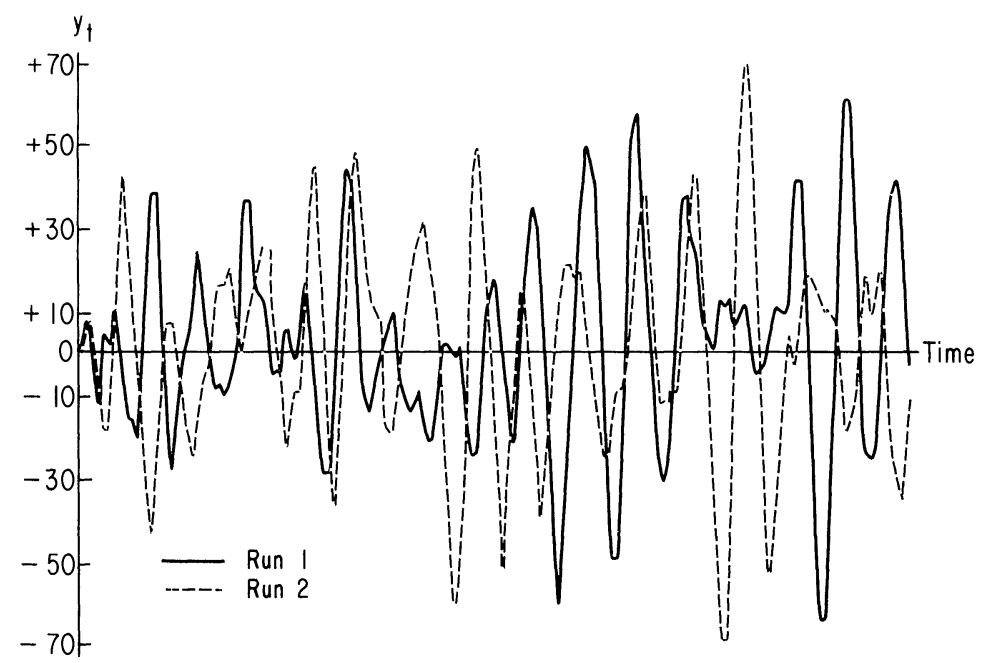

FiguRE 5.-Comparison of two series $\left\{y_{t}^{(1)}\right\}$ and $\left\{y_{t}^{(2)}\right\}$ generated by runs 1 and 2 respectively of the Samuelson-Hicks model. 
generated by run 2 . We recall that the only difference between run 1 and run 2 lies in the fact that we use a different starting value for the sequence of pseudorandom numbers used to generate the stochastic variate $w_{t}$. In other words, we know beforehand that the theoretical spectra of runs 1 and 2 are identical, i.e., $P=1$. Figure 5 shows the simulated time paths of the two relevant series $\left\{y_{t}^{(1)}\right\}$ and $\left\{y_{t}^{(2)}\right\}$. A cursory glance at Figure 5 might lead one to conclude that the two series have little in common and are the result of the use of two different economic policies. Despite their apparent dissimilarity when inspected visually, the two series have been generated in exactly the same manner (i.e., the factor loadings are identical), with one exception-namely, different starting values were used for the random number generator which supplies the stochastic term $w_{t}$.

We now bring a measure of order to the two series by constructing a 95 per cent simultaneous confidence band for $P$ in Figure 6. As expected, the horizontal line $P=1$ corresponding to the true ratio lies within the confidence band for all values of $(0 \leqslant \omega \leqslant \pi)$.

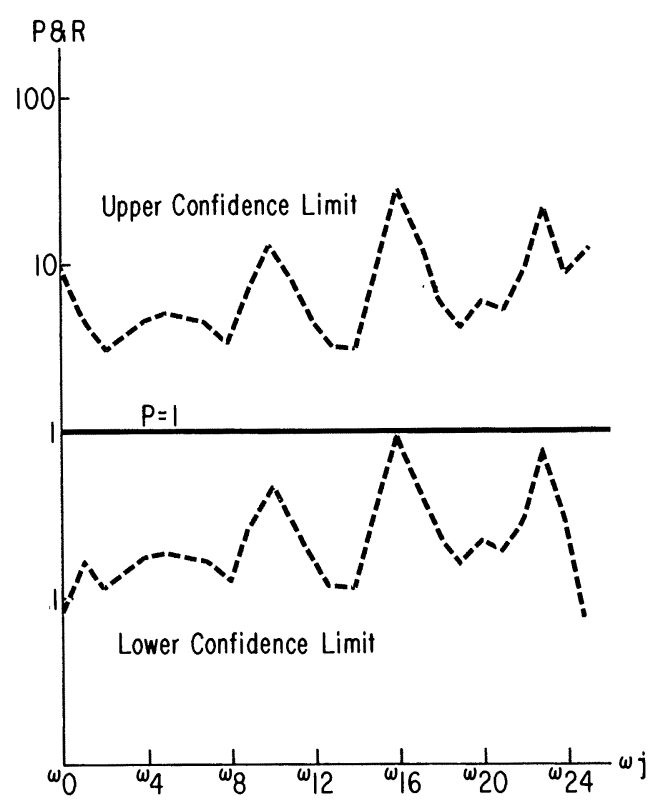

FIGURE 6.-Simultaneous confidence band for comparing the empirical spectra $f_{1}(\omega)$ and $f_{2}(\omega)$ of two series $\left\{y_{t}^{(1)}\right\}$ and $\left\{y_{t}^{(2)}\right\}$ generated by runs 1 and 2 respectively of the Samuelson-Hicks model.

For a second example, we compare the time path of national income generated by run 1 with that of run 3 . Recall that run 3 represents the result of a change in monetary policy (accelerator coefficient) and a change in fiscal policy (governmental parameter). Constructing a 95 per cent simultaneous confidence band for $P$ in Figure 7 we observe that the graph of the horizontal line $P=1$ falls outside of the confidence band at $\omega_{1}, \omega_{2}, \omega_{5}, \omega_{20}, \omega_{22}$, and $\omega_{24}$ thus showing that the 
spectra are indeed different. As expected, the true ratio $P$, computed using formula (41) lies within the simultaneous confidence band, except at some high frequencies $\left(\omega_{20}, \omega_{22}, \omega_{24}\right)$, where "window leakage" [4] from the lower frequencies affects these weaker, and less important, frequencies. Figure 7 demonstrates even more specifically that the spectral shapes of run 1 and run 3 are different, because no horizontal line can be drawn which lies within the confidence band. If the spectral shapes were the same, i.e., if $f_{1}(\omega)=k f_{3}(\omega)$, for some $k$, then the ratio $P$ would equal $k$ and its graph would be a horizontal line.

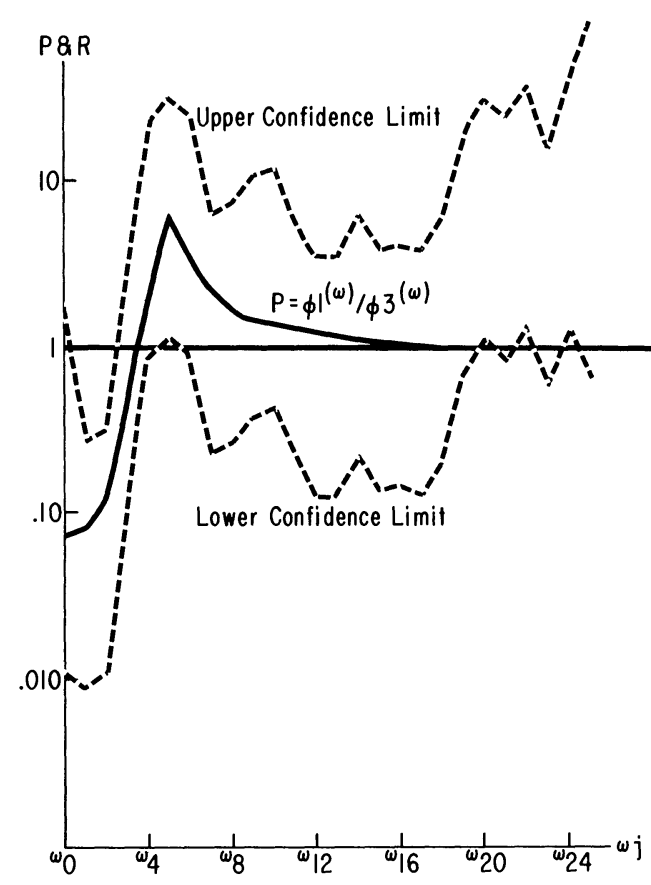

FIGURE 7.- Simultaneous confidence band for comparing the empirical spectra $f_{1}(\omega)$ and $f_{3}(\omega)$ of two series $\left\{y_{t}^{(1)}\right\}$ and $\left\{y_{t}^{(3)}\right\}$ generated by runs 1 and 3 respectively of the Samuelson-Hicks model.

Thus far we have been concerned with the use of spectral analysis to decompose the variance of a time series into its frequency components [44]. We now consider the variance as a whole for a given time series. The natural estimator of $\sigma^{2}$ is of course the sample variance $s^{2}$. We would, therefore, hope to use classical $\chi^{2}$ or $F$-ratio statistics to analyze the variance of a given time series or two or more series. Because the time series is autocorrelated, however, $s^{2}$ does not generally have a $\chi^{2}$ distribution with $(n-1)$ degrees of freedom. In a sense, the autocorrelation of the observations reduces the amount of information they provide about the variance, so that the number of degrees of freedom for $s^{2}$ is less than $(n-1)$. In fact, if the observations $x_{t}$ are highly autocorrelated as in a pure sine wave, then $s^{2}$ has only two degrees of freedom [4, p. 21-25]. To determine in 
general the number of degrees of freedom for $s^{2}$, we resort to its spectral decomposition.

We begin by noting that equation (36) gives the theoretical variance $\sigma^{2}$ in terms of the spectrum $\phi$. Similarly, the sample variance $s^{2}$ is given in terms of the estimated spectrum $f$ :

$$
s^{2}=c_{0}=\frac{1}{m}\left[\frac{f(0)}{2}+\sum_{j=1}^{m-1} f\left(\omega_{j}\right)+\frac{f(\pi)}{2}\right] .
$$

This formula requires that the weight $\lambda_{0}$ in equation (37) be unity, a condition satisfied by every reasonable spectrum estimator.

Thus $s^{2}$ is displayed as the sum of somewhat ${ }^{12}$ dependent terms, each of which is a multiple of $\chi^{2}$ on $2 n / m$ degrees of freedom. Following a technique developed by Blackman and Tukey $\left[4\right.$, p. 24], it can be shown that $s^{2}$ essentially ${ }^{13}$ follows a $\chi^{2}$ distribution with degrees of freedom approximated by

$$
k=\frac{\left[\frac{f(0)}{2}+\sum_{j=1}^{m-1} f\left(\omega_{j}\right)+\frac{f(\pi)}{2}\right]^{2}}{\frac{[f(0)]^{2}}{2}+\sum_{j=1}^{m-1}\left[f\left(\omega_{j}\right)\right]^{2}+\frac{[f(\pi)]^{2}}{2}} \times \frac{n}{m} .
$$

We note in passing that if the spectrum $f(\omega)$ is constant (corresponding to uncorrelated observations), then $k$ reduces to $n$, which is approximately the correct value $n-1$. Depending on the degree to which $f(\omega)$ is not constant (correlated observations), $k$ will be reduced. Now that the distribution of $s^{2}$ is known, we can test $\sigma^{2}$ or construct confidence intervals for one series alone or a two series comparison.

For example, to construct a confidence interval for $\sigma_{1}^{2} / \sigma_{2}^{2}$, the ratio of variances of two series, we note that

$$
F=\frac{n_{1} s_{1}^{2} / \sigma_{1}^{2} k_{1}}{n_{2} s_{2}^{2} / \sigma_{2}^{2} k_{2}}
$$

has an $F$ (variance-ratio) distribution with $k_{1}, k_{2}$ degrees of freedom. If we let $F_{k_{1}, k_{2}, 975}$ and $F_{k_{1}, k_{2}, 025}$ denote the percentiles which leave 2.5 per cent of the probability in the lower and upper tails respectively, then

$$
\operatorname{Pr}\left(F_{k_{1}, k_{2}, .975}<\frac{n_{1} s_{1}^{2} / \sigma_{1}^{2} k_{1}}{n_{2} s_{2}^{2} / \sigma_{2}^{2} k_{2}}<F_{k_{1}, k_{2}, .025}\right)=.95 .
$$

Solving for $\sigma_{1}^{2} / \sigma_{2}^{2}$, we obtain our desired confidence interval :

$$
\operatorname{Pr}\left(\frac{n_{1} s_{1}^{2} k_{2}}{n_{2} s_{2}^{2} k_{1}} \frac{1}{F_{k_{1}, k_{2}, .025}}<\sigma_{1}^{2} / \sigma_{2}^{2}<\frac{n_{1} s_{1}^{2} k_{2}}{n_{2} s_{2}^{2} k_{1}} \frac{1}{F_{k_{1}, k_{2}, .975}}\right)=.95 \text {. }
$$

\footnotetext{
${ }^{12}$ Blackman and Tukey [4] have shown that adjacent spectral estimates are correlated, but that nonadjacent estimates are practically uncorrelated.

${ }^{13}$ Strictly speaking, both here and in every other occurrence, it is $n s^{2} / \sigma^{2}$ which has a $\chi^{2}$ distribution and not $s^{2}$.
} 
For the comparison of runs 1 and 2, the actual 95 per cent confidence interval was computed to be

$$
.429<\sigma_{1}^{2} / \sigma_{2}^{2}<1.72
$$

which contains the true value, unity.

\section{SPECTRAL ANALYSIS: A LARGER ROLE?}

In the foregoing text, we have employed spectral analysis in conjunction with an econometric model, albeit primitive. Not all writers would cast spectral (and cross spectral) analysis into so modest a role. Granger and Hatanaka would apparently have these techniques become the primary guides in reshaping and restating much economic theory, thus turning methodological biases away from "classical" model building and toward more "advanced" modes of time series analysis. The present authors do not now champion the exclusiveness of either model building or spectral techniques, but would insist that benefits can be derived from their joint and complementary use.

In addition to the outlets for compromise that are proposed in the sections above, it is important to bring this same notion to the issue of prediction. One sometimes hears that whereas econometric models can generate forecasts and that knowledge of the power spectrum cannot, the latter is of little relevance to forecasters. According to this argument one would also be disposed to claim that it is the shovel that turns the earth, not man's understanding of cultivation, and therefore the latter is immaterial to farmers. Working from a different and more satisfactory premise - namely, how something is done affects what is donewe could contend that the spectral technique, as a tool of validation, may be pertinent to forecasters. For when an econometric model generates a series for a variable, and that series yields a power spectrum which is fundamentally different than the spectrum of the actual data, ${ }^{14}$ then the simulator learns that his model is defective at least along this dimension. What weight is to be attached to such information cannot be dictated, but an out-of-hand value of zero seems unwarranted. Until a model's predictative ability can be judged against actual data in future periods, its performance on tests of validation over past periods will certainly determine to a great extent how much confidence is to be given to the predicted levels, impact multipliers, and so on, which it currently produces.

\section{SUMMARY}

In this paper, we have attempted to demonstrate the potential value of spectral analysis as a technique for analyzing data generated by computer simulation experiments with econometric models. By constructing the appropriate confidence bands, spectral analysis enables one to validate the output of simulation experiments and to compare the results of alternative economic policies associated with different series generated by an econometric model.

${ }^{14}$ Subject, of course, to the problems of sample size and nonstationarity. 
The application of spectral analysis to series generated by computer simulation experiments differs from the application of spectral analysis to "real world" economic series in two ways. First, with computer simulation we are not subject to the same type of sample size limitations that one encounters with economic time series. Second, with computer simulation we can sample across the ensemble, an alternative that is rarely available with actual economic series. As of yet, this opportunity has not been exploited by economists or simulators.

\section{Duke University}

\section{REFERENCES}

[1] Adelman, Irma, and Frank L. Adelman: "The Dynamic Properties of the Klein-Goldberger Model," Econometrica, XXVII (October, 1959), pp. 596-625.

[2] Adelman, Irma: "Long Cycles-A Simulation Experiment," Symposium on Simulation Models. Austin C. Hogatt and Frederick E. Balderston (editors), Cincinnati: South-western Publishing Co., 1963.

[3] Bartlett, M. S.: An Introduction to Stochastic Processes with Special Reference to Methods and Applications. Cambridge: The University Press, 1962.

[4] Blackman, R. B., and J. W. Tukey: The Measurement of Power Spectra. New York: Dover Publications, Inc., 1958.

[5] Burdick, Donald S., and Thomas H. Naylor: "Design of Computer Simulation Experiments for Industrial Systems," Communications of the ACM, IX (May, 1966), pp. 329-339.

[6] Cunnyingham, J.: "Spectral Analysis of Economic Time Series," Working Paper No. 16, U.S. Department of Commerce, Bureau of the Census, New York, 1963.

[7] De Leeuw, Frank: "Financial Markets in Business Cycles: A Simulation Study," American Economic Review, LIV (May, 1964). pp. 309-323.

[8] Duesenberry, James S., Otto Eckstein, and Gary Fromm: "A Simulation of the United States Economy in Recession,” Econometrica, XXVIII (October, 1960), pp. 749-809.

[9] Duesenberry, J. S., G. Fromm, L. R. Klein, And E. Kun: The Brookings Quarterly Econometric Model of the United States. Chicago: Rand McNally \& Co., 1965.

[10] Fishman, George S., AND Philip J. Kiviat: "The Analysis of Simulation-Generated Time Serics," Management Science, XIII (March, 1967), pp. 525-557.

[11] FraSer, D. A. S.: Nonparametric Methods in Statistics. New York: John Wiley \& Sons, 1957.

[12] Fromm, Gary: "Recent Monetary Policy: An Econometric View," National Banking Review, III (March, 1966), pp. 299-306.

[13] Granger, C. W. J.: "The Typical Spectral Shape of an Economic Variable," Econometrica, XXXIV (January, 1966), pp. 150-161.

[14] Granger, C. W. J., and M. Hatanaka: Spectral Analysis of Economic Time Series. Princeton, N.J.: Princeton University Press, 1964.

[ $\rightarrow$ Granger, C. W. J., AND O. Morgenstern: "Spectral Analysis of Stock Market Prices," Kyklos, XVI (1963), pp. 1-27.

[16] Grenander, U., AND M. Rosenblatt : Statistical Analysis of Stationary Time Series. New York: John Wiley \& Sons, 1957.

[17] Gross, Donald, and JaCk L. RaY: "A General Purpose Forecast Simulator," Management Science, XI (April, 1965), pp. 119-135.

[18] Hannan, E. J.: Time Series Analysis. New York: John Wiley \& Sons, 1960.

[19] — : "Regression for Time Series," Proceedings of the Symposium on Time Series Analysis. Murray Rosenblatt (editor). New York: John Wiley \& Sons, 1962.

[20] HatanaKa, M.: "A Spectral Analysis of Business Cycle Indicators: Lead-Lag in Terms of All Time Points," Econometric Research Program, Princeton, Research Memorandum No. 53, 1963.

[21] Hickman, Bert G. (editor): Quantitative Planning of Economic Policy. Washington, D.C.: The Brookings Institution, 1965. 
[22] Hicks, J. R.: A Contribution to the Theory of the Trade Cycle. Oxford: Clarendon Press, 1950.

[23] Howrey, E. Philip: "Stabilization Policy in Linear Stochastic Systems," unpublished paper, Econometric Research Program, Princeton University, January, 1966 (mimeographed).

[24] Jenkins, G. M.: "General Considerations in the Analysis of Spectra," Technometrics, III (May, 1961), pp. 133-166.

[25] Jenkins, G. M., AND M. B. Priestly: "The Spectral Analysis of Time Series," Journal of the Royal Statistical Society, B, XIX (1957), pp. 1-12.

[26 Karreman, Herman F.: "Computer Programs for Spectral Analysis of Economic Time Series," Princeton University, Econometric Research Program, Research Memorandum No. 59, July $15,1963$.

[27] Kendall, M. G.: “On Autoregressive Time Series," Biometrika, XXXIII (August, 1944), pp. 105-122.

[28] LEE, Y. W.: Statistical Theory of Communications. New York: John Wiley \& Sons, 1960.

[29] LiU, TA-Chung: "An Exploratory Quarterly Econometric Model of Effective Demand in the Postwar U.S. Economy," Econometrica, XXXI (July, 1963), pp. 301-348.

[30] Malinvaud, E.: Statistical Methods of Econometrics. Chicago: Rand McNally, 1966.

[31] Naylor, Thomas H., Joseph L. Balintfy, Donald S. BURdick, and Kong Chu: Computer Simulation Techniques. New York: John Wiley \& Sons, 1966.

[32] Naylor, Thomas H., Donald S. Burdick, and W. Earl Sasser: "Computer Simulation Experiments with Economic Systems: The Problem of Experimental Design," Journal of the American Statistical Association (Dec., 1967).

[33] Naylor, ThOmas H., AND J. M. Finger: "Verification of Computer Simulation Models," Management Science (Oct., 1967).

[34] Naylor, Thomas H., William H. Wallace, and W. Earl Sasser: "A Computer Simulation Model of the Textile Industry," Journal of the American Statistical Association (Dec., 1967).

[35] Naylor, Thomas H., Kenneth Wertz, and Thomas Wonnacott: "Methods for Analyzing Data from Computer Simulation Experiments," Communications of the ACM (Nov., 1967).

[36] — : "Some Methods for Evaluating the Effects of Economic Policies Using Simulation Experiments," Review of the International Statistical Institute (Feb., 1968).

[37] Nerlove, M.: "Spectral Analysis of Seasonal Adjustment Procedures," Econometrica, XXXII (1964), pp. 241-286.

[38] - Spectral Comparisons of Two Seasonal Adjustment Procedures, Technical Report No. 2, National Science Foundation Grant GS-142, June 1, 1964.

[39] Parzen, Emanuel: "Mathematical Considerations in the Estimation of Spectra," Technometrics, III (May, 1961), pp. 167-190.

[40] : Stochastic Processes. San Francisco: Holden-Day, 1962.

[41] Quenouille, M. H.: The Analysis of Multiple Time-Series. New York: Hafner Publishing Co., 1957.

[42] Rosenblatt, Murray: Proceedings of the Symposium on Time Series Analysis. New York: John Wiley \& Sons, 1962.

[43] Samullson, Paul A.: "Interactions Between the Multiplier Analysis and the Principle of Acceleration," Review of Economic Statistics, XII (May, 1938), pp. 75-78.

[44] Tukey, JohN W.: "Discussion, Emphasizing the Connection Between Analysis of Variance and Spectrum Analysis," Technometrics, III (May, 1961), pp. 191-219.

[45] WIENER, NORBERT: Extrapolation, Interpolation, and Smoothing of Stationary Time Series. Cambridge: The M.I.T. Press, 1949.

[46] Wonnacott, Thomas: "Spectral Analysis Combining a Bartlett Window With an Associated Inner Window," Technometrics, III (May, 1961), pp. 237-245. 